\title{
Study of Memory Effect in a Fuzzy EOQ Model with No Shortage
}

\author{
Rituparna Pakhira \\ Department of Applied Mathematics, University of Calcutta, Kolkata, 700009, West Bengal, India \\ E-mail: rituparna.pakhira@gmail.com \\ Uttam Ghosh and Susmita Sarkar \\ Department of Applied Mathematics, University of Calcutta, Kolkata, 700009, West Bengal, India \\ E-mail: uttam@yahoo.com, susmita62@yahoo.co.in
}

Received: 09 April 2019; Accepted: 12 August 2019; Published: 08 November 2019

\begin{abstract}
The feature of the fractional order derivative and fractional order integration is one of the important tools to realize the beauty of the fractional calculus. Fractional order derivative and integration has a long history like classical calculus but its users are much less compared to the classical calculus. The purpose of this paper is to study an inventory model with linear type demand rate under the fuzzy environment. This paper also wants to introduce the memory effect property of fractional order derivative which can help to setup the model more authentic. Two advantages have been included to the model (i) memory effect,(ii) fuzzy environment. Here, the fractional order model is defuzzyfied using (i) signed distance method,(ii) graded mean integration method. Fuzzification can close to the reality with incorporating uncertainty behavior of some economic parameters of the inventory system and fractional order can explain the memory phenomena. For this problem due to illustrate defuzzification, set up cost, holding cost per unit, per unit cost are assumed as triangular fuzzy numbers. Fractional order derivative and integration are applied to develop the whole work. It is known that fractional calculus is a valuable tool to describe memory phenomena. Fractional order is established as the index of the memory. In this paper, depending on strength of memory, memory phenomena considered in two steps(i) long memory,(ii) short memory. The proposed fuzzy models and technique lastly have been illustrated. Results of two defuzzyfications are compared with graphical presentations. This present studies can help to moderate the classical fuzzy inventory model. From the numerical studied it is observed that in long memory effect, profit is good compared to the low memory effect or memory less system.
\end{abstract}

Index Terms - Fractional order derivative, Fractional order inventory model under fuzzy environment, Long memory effect, Short memory effect.

\section{INTRODUCTION}

For a real situation of the business, market changes rapidly. Due to the fact, some parameters and variables are highly uncertain. In such situations, these parameters and variables are described as fuzzy parameters. The fuzzification declares authenticity to the model by allowing vagueness in the whole cost parameters which brings it closer to reality. We want to generalize the concept of the paper D.Sharmitaetal[18] using fractional calculus on the basic EOQ model. Here, fractional calculus has been applied instead of ordinary calculus. Fractional order derivative and fractional order integration is used to include non-locality of the system. Fractional calculus are becoming a favourite research topic to the different field researchers.

Recently, the application of fractional order integration and differentiation becomes a hot research topic. Fractional integration and fractional differentiation are generalizations of notions of integer-order integration and differentiation and it can include $n$-th derivatives and $n$ fold integrals. Physical interpretations of any mathematical theory can close to reality and help to connect to the real world problem. Fractional calculus has some advantage from the ordinary calculus. For example, some properties of fractional order derivative can include the violation of Leibnitz rule and violation of chain rule. Some unusual properties [21] of the fractional order derivatives describe complex properties of the chain rules. The physical interpretation of the fractional calculus is that fractional order of fractional derivative and integration is an index of memory $[1,9,10,11]$. Using the concept of memory several research papers have been developed [1,2,3,4,5,6,7]. Some of them are discussed shortly. Application of memory effect using fractional calculus was done by M.Saedianetal[1] in the biological model. Besides, Tarasovaetal[2,3,4,5,6,7] have developed many research article using the concept of memory effect to the economic models. Memory 
dependent inventory models [9,10,11,23,24,20] have been developed by Pakhira et al to establish memory effect in EOQ models with different terms and conditions. Different memory dependent inventory models[9,10,11,23,24,20] have been developed in different articles without fuzzy environment.

Due to the above fact, we want to incorporate here fuzzy environment. In this paper, our aim is to develop a memory dependent EOQ model under the fuzzy environment using fractional calculus. Some researchers have worked on the Classical inventory model $[17,18]$ under the fuzzy environment with assuming different conditions on the cost parameters. Some of them are discussed shortly. As for example, Majumdar.P etal[17] developed an EPQ model of deteriorating items under partial trade credit financing and demand declining market in crisp and fuzzy environment. N.K.Mandal[18] developed a Fuzzy economic order quantity model with ranking fuzzy cost parameters. D. Sharmitaetal[18] established an inventory model for deteriorating items with shortages and exponential demand involving fuzzy environment. Besides, Dutta.Det al also developed a fuzzy inventory model for deteriorating items with shortages under fully backlogged condition. Different above mentioned authors do not use the beauty of fractional calculus in their model. Fractional has a beautiful property.

In this paper, this analogy 'fractional order as the index of memory' has been used to illustrate the model. Fractional order inventory model has been developed to include memory effect under the fuzzy environment. The fractional order model is also fuzzified using Graded mean integration method and signed distance method. Since the fuzzy environment can close to reality with incorporating uncertainty behavior to the system. Inventory level with respect to time has been drawn to show the changing behavior for (i) long memory,(ii)short memory,(iii) memory less system. Then we want to discuss the rearrangement part of the paper to overview easily.

The rest of the paper is organized as follows: In section III, the preliminary introduction of fractional calculus are introduced. Section IV (C )is about the formulation of the classical inventory model. In section $\mathrm{V}$, we have discussed the procedure of fractionalization of the classical model. In section VI, Mathematical formulation of the fractional order inventory model is discussed. Fuzzy fractional order inventory model has been given in the section-VII, Numerical examples in the section-VIII, Graphical presentations are done in the section-IX. Lastly, conclusions and future research works are discussed in the section-X.

\section{RELATED DEVELOPED WORK}

The classical fuzzy inventory model has been described in the paper[18,22].This paper is followed from this two papers[18,22].The Memory concept has been followed from the different papers like[10,11,20,21,23,24].

\section{REVIEW OF FRACTIONAL CALCULUS}

To develop the model, Caputo fractional order derivative and fractional Laplace transform method has been used, are discussed shortly.

\section{A. Euler Gamma Function}

Euler's gamma function is one of the best tools in fractional calculus which was proposed by the Swiss mathematicians Leonhard Euler (1707-1783).The gamma function $\Gamma(x)$ is continuous extension from the factorial notation. The gamma function is denoted and defined by the formulae

$$
\Gamma(x)=\int_{0}^{\infty} t^{(x-1)} e^{-t} d t \quad x>0
$$

$\Gamma(x)$ is extended for all real and complex numbers and the gamma function satisfies some basic properties

$$
\left.\begin{array}{l}
\Gamma(x+1)=x \Gamma(x) \Rightarrow \Gamma(x)=\frac{\Gamma(x+1)}{x}, \\
\Gamma\left(\frac{1}{2}\right)=\frac{\sqrt{\pi}}{2}, \Gamma\left(-\frac{7}{6}\right)=-\frac{7}{6} \Gamma\left(\frac{1}{6}\right) \\
\Gamma\left(-\frac{8}{9}\right)=-\frac{8}{9} \Gamma\left(\frac{1}{9}\right), \Gamma\left(-\frac{7}{8}\right)=-\frac{8}{7} \Gamma\left(\frac{1}{8}\right) \cdot
\end{array}\right\}
$$

Numerically $x$ ! can be evaluated for all positive integer values numerically but $\Gamma(x+1)$ can be evaluated for real values.

\section{B. Riemann-Liouville fractional derivative $(R-L)$}

Left Riemann-Liouville fractional derivative[12-13] of order $\alpha$ is denoted and defined as follows

$$
{ }_{a} D_{x}^{\alpha}(f(x))=\frac{1}{\Gamma(m-a)}\left(\frac{d}{d x}\right)^{m} \int_{a}^{x}(x-\tau)^{(m-\alpha-1)} f(\tau) d \tau
$$

where $x>0$

Right Riemann-Liouville fractional derivative of order $\alpha$ is defined as follows

$$
{ }_{x} D_{b}^{\alpha}(f(x))=\frac{1}{\Gamma(m-a)}\left(-\frac{d}{d x}\right)^{m} \int_{x}^{b}(\tau-x)^{(m-\alpha-1)} f(\tau) d \tau
$$

where $x>0$

Riemann-Liouville fractional derivative of any constant function is not equal to zero which creates a distance between ordinary calculus and fractional calculus.

\section{Caputo fractional order derivative}

Left Caputo fractional derivative [14] for the function $f(x)$ which has continuous, bounded derivatives in 
$[a, b]$ is denoted and defined as follows

$$
\begin{aligned}
& { }_{a}^{C} D_{x}^{\alpha}(f(x))=\frac{1}{\Gamma(m-\alpha)} \int_{\alpha}^{x}(x-\tau)^{(m-\alpha-1)} f^{m}(\tau) d \tau \\
& \text { where } 0 \leq m-1<\alpha<m
\end{aligned}
$$

Right Caputo fractional derivative for the function $f(x)$ which has continuous and bounded derivatives in $[a, b]$, is defined as follows

$$
\begin{aligned}
& { }_{x}^{C} D_{b}^{\alpha}(f(x))=\frac{1}{\Gamma(m-\alpha)} \int_{x}^{b}(\tau-x)^{(m-\alpha-1)} f^{m}(\tau) d \tau \\
& \text { where } 0 \leq m-1<\alpha<m
\end{aligned}
$$

$$
{ }_{a}^{C} D_{x}^{\alpha}(A)=0, \text { where } A=\text { constant } .
$$

\section{Fractional Laplace transforms Method}

The Laplace transform of the function $f(t)$ is defined as

$$
F(s)=L(f(t))=\int_{0}^{\infty} e^{-s t} f(t) d t
$$

where $\mathrm{s}>0$ and $s$ is called the transform parameter. The Laplace transformation of $n^{\text {th }}$ order derivative is defined as

$$
L\left(f^{n}(t)\right)=s^{n} F(s)-\sum_{k=0}^{n-1} s^{n-k-1} f^{k}(0)
$$

where $f^{n}(t)$ denotes $n^{\text {th }}$ derivative of the function $f$ with respect to $t$ and for non - integer $m$ it is defined in generalized form[8-9] as,

$$
L\left(f^{m}(t)\right)=s^{m} F(s)-\sum_{k=0}^{n-1} s^{k} f^{m-k-1}(0)
$$

Where, $(n-1)<m \leq n$.

In the next sections, we first discuss the classical inventory model which can help to provide a link to

\begin{tabular}{|c|c|}
\hline (i) $D(t)$ : Demand rate & $\begin{array}{l}\text { (ii) } Q: \text { Total order } \\
\text { quantity }\end{array}$ \\
\hline (iii) $U$ : Per unit cost & $\begin{array}{l}(i v) C_{1}: \text { Inventory } \\
\text { holding cost per unit }\end{array}$ \\
\hline $\begin{array}{l}(v) C_{3} \text { : Ordering cost or setup } \\
\text { cost }\end{array}$ & $\begin{array}{l}(v i) I(t): \text { Stock level } \\
\text { or inventory level }\end{array}$ \\
\hline (vii) $T$ : Ordering interval & $\begin{array}{l}\text { (viii) } H C_{\alpha, \beta}: \text { Inventory } \\
\text { holding cost per } \\
\text { cycle for the } \\
\text { fractional order } \\
\text { inventory model }\end{array}$ \\
\hline $\begin{array}{l}(i x) T_{\alpha, \beta}^{G^{*}}: \text { Optimal ordering } \\
\text { interval using Graded Mean } \\
\text { integration method }\end{array}$ & $\begin{array}{l}(x) T C_{\alpha, \beta}^{a v G}: \text { Total } \\
\text { average cost using } \\
\text { graded mean } \\
\text { integration method. }\end{array}$ \\
\hline 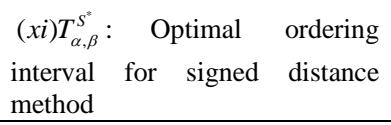 & $\begin{array}{l}(x i i)(B, .),(\Gamma, .): \text { Beta } \\
\text { function and gamma } \\
\text { function respectively }\end{array}$ \\
\hline $\begin{array}{l}\text { (xiii)TC } C_{\alpha, \beta}^{S^{*}}: \text { Minimized total } \\
\text { average cost during the total } \\
\text { time interval }[0, T] \text { using signed } \\
\text { distance method }\end{array}$ & $\begin{array}{l}(x i v) T C_{\alpha, \beta}^{G^{*}}: \text { Minimized total } \\
\text { average cost during the total } \\
\text { time interval }[0, T] \text { using } \\
\text { graded mean integration } \\
\text { method }\end{array}$ \\
\hline $\begin{array}{l}(x v) T C_{\alpha, \beta}^{a v S}: \text { Total average cost } \\
\text { during the total time interval } \\
\text { using signed distance integration } \\
\text { method }\end{array}$ & \\
\hline
\end{tabular}
establish the fractional order inventory model.

\section{ClassicAl InVEnTORY MODEL}

The classical model has been developed in this section.

\section{A. Notations}

The notations are given to develop the inventory model.
Table 1. Different symbols and items for the model.

Next, we want to discuss the assuming some assumptions to develop the model.

\section{B. Assumptions}

The below assumptions are made to develop the model. (i) Lead time is zero,(ii)Time horizon is infinite,(iii)There is no shortage,(iv)There is no deterioration.(v) Demand rate is $D(t)=(a+b t)$ for $0 \leq t \leq T$. Then, we have discussed the classical inventory model formulation.

\section{Classical inventory model Formulation}

During the total period $[0, T]$, the inventory level depletes due to the demand rate $(a+b t), a, b>0$ where the shortage is not allowed. Hence, the classical model is governed in by the ordinary differential equation as

$$
\frac{d(I(t))}{d t}=-(a+b t) \text { for } 0 \leq t \leq T
$$

with boundary conditions are $I(t)=0$ and $I(0)=Q$. Inventory level is obtained by solving (10) with the boundary condition $I(t)=0$ in the following form

$$
I(t)=a(T-t)+\frac{b}{2}\left(T^{2}-t^{2}\right)
$$

Using initial condition $I(0)=Q$, the optimal order quantityis obtained as, 


$$
Q=\left(a T+\frac{b}{2} T^{2}\right)
$$

Corresponding total inventory holding cost over the time interval $[0, T]$ is denoted by $H C(T)$ and defined as follows

$$
\begin{aligned}
H C(T) & =C_{1} \int_{0}^{T}\left(a(T-t)+\frac{b}{2}\left(T^{2}-t^{2}\right)\right) d t \\
& =C_{1}\left(\frac{a T^{2}}{2}+\frac{b T^{3}}{3}\right)
\end{aligned}
$$

$C_{1}$ is the holding cost per unit.

Hence, the total cost is as

$$
\begin{gathered}
T C(T)=\text { Purchasing cost }(P C)+\text { holding } \operatorname{cost}(H C(T) \\
\quad+\text { set up cost }\left(C_{3}\right) \\
\therefore T C(T) \\
=\left(U\left(a T+\frac{b}{2} T^{2}\right)+C_{1}\left(\frac{a T^{2}}{2}+\frac{b T^{3}}{3}\right)+C_{3}\right)
\end{gathered}
$$
is

Therefore, the total average cost per unit time per cycle

$$
\begin{aligned}
& \operatorname{TOC}^{a v}(T) \\
& =\frac{\left(U\left(a T+\frac{b}{2} T^{2}\right)+C_{1}\left(\frac{a T^{2}}{2}+\frac{b T^{3}}{3}\right)+C_{3}\right)}{T}
\end{aligned}
$$

Thus, the objective of the classical EOQ model can be represented in the form as,

$$
\left\{\begin{array}{l}
\operatorname{MinTC}(T)=\frac{\left(U Q+H C(T)+C_{3}\right)}{T} \\
\text { Subject to } T \geq 0
\end{array}\right.
$$

In the next section, we have developed the fractional order model with memory kernel.

\section{FRACTIONAL ORDER INVENTORY MODEL WITH MEMORY KERNEL}

To study the influence of memory effects, first the differential equation (10) is written using the memory kernel function in the following form [1].

$$
\frac{d I(t)}{d t}=-\int \mathrm{k}\left(t-t^{\prime}\right)\left(a+b t^{\prime}\right) d t^{\prime}
$$

in which $k\left(t-t^{\prime}\right)$ plays the role of a time-dependent kernel. For Markov process it is equal to the delta function $\delta\left(t-t^{\prime}\right)$ that generates the equation (10).In fact, any arbitrary function can be replaced by a sum of delta functions, thereby leading to a given type of time correlations. This type of kernel promises the existence of scaling features as it is often intrinsic in most natural phenomena. Thus, to generate the fractional order model we consider $k\left(t-t^{\prime}\right)=\frac{1}{\Gamma(1-\alpha)}\left(t-t^{\prime}\right)^{\alpha-2}, \quad$ where $0<\alpha \leq 1$ and $\Gamma(\alpha)$ denotes the gamma function.Using the definition of fractional derivative [2], the equation (17) can be written to the form of fractional differential equations with the Caputo-type derivative in the following form as,

$$
\frac{d I(t)}{d t}=-{ }_{0} D_{t}^{-(\alpha-1)}(a+b t)
$$

Now, applying fractional Caputo derivative of order $(\alpha-1)$ on both sides of (18), and using the fact that Caputo fractional order derivative and fractional integral are inverse operators, the following fractional differential equations can be obtained for the model

$$
\begin{gathered}
{ }_{0}^{C} D_{t}^{\alpha}(I(t))=-(a+b t) \text { or equivalently } \\
\frac{d^{\alpha}(\mathrm{I}(\mathrm{t}))}{d t^{\alpha}}=-(a+b t) \\
\text { for } 0<\alpha \leq 1.0,0 \leq t \leq T
\end{gathered}
$$

with boundary conditions $I(T)=0$ and $I(0)=Q$.

Depending on the range of $\alpha$, long memory effect and short memory effect has been defined.

Long and Short Memory Effect: The strength of memory depends by the order of the fractional order derivative and fractional order integration. If the fractional order index $\alpha$ is in $(0,0.5]$ then we define the system as the long memory affected system and for short memory effected system $\alpha$ is in $(0.5,1]$.

In the next section mathematical solution and analytical results are discussed due to reach the final numerical result.

\section{MATHEMATICAL Formulation OF THE FraCtionAL ORDER INVENTORY MODEL}

Here, we consider the fractional order inventory model which will be solved by using Laplace transform method with the boundary condition $I(T)=0$. In operator form the fractional differential equation of (19) can be represented as 


$$
\begin{aligned}
& D^{\alpha}(I(t))=-(a+b t), \\
& D^{\alpha} \equiv \frac{d^{\alpha}}{d t^{\alpha}}, \text { with } I(T)=0
\end{aligned}
$$

where the operator $D^{\alpha}$ stands for the Caputo fractional order derivative with the operator

$$
\left(D^{\alpha}={ }_{0}^{c} D_{t}^{\alpha}\right) \text {. }
$$

Solving the above equation (20), we get the required inventory level with fractional effect

$$
I(t)=\left(\frac{a\left(T^{\alpha}-t^{\alpha}\right)}{\Gamma(1+\alpha)}+\frac{b\left(T^{\alpha+1}-t^{\alpha+1}\right)}{\Gamma(\alpha+2)}\right)
$$

For $\alpha=1.0$, the equation (21) becomes to the equation (11).

Using the boundary condition $I(T)=0$ on the equation (21), the total order quantity is obtained as

$$
I(0)=Q=\left(\frac{a\left(T^{\alpha}\right)}{\Gamma(1+\alpha)}+\frac{b\left(T^{1+\alpha}\right)}{\Gamma(\alpha+2)}\right)
$$

and corresponding the inventory level at time $t$ being,

$$
I(t)=\left(\frac{a\left(T^{\alpha}-t^{\alpha}\right)}{\Gamma(1+\alpha)}+\frac{b\left(T^{\alpha+1}-t^{\alpha+1}\right)}{\Gamma(\alpha+2)}\right)
$$

Purchasing cost is as

$$
P C=U \times Q=U\left(\frac{a\left(T^{\alpha}\right)}{\Gamma(1+\alpha)}+\frac{b\left(T^{1+\alpha}\right)}{\Gamma(\alpha+2)}\right)
$$

(where $U$ is the per unit cost)

For the model (19), the $\beta-(0<\beta \leq 1)$ order total inventory holding cost is denoted as $H C_{\alpha, \beta}(T)$ and defined as

$$
\begin{aligned}
H C_{\alpha, \beta}(T)= & C_{10} D_{T}^{-\beta}(I(t)) \\
= & \frac{C_{1}}{\Gamma(\beta)} \int_{0}^{T}(T-t)^{(\beta-1)}(I(t)) d t \\
= & \frac{C_{1} a T^{(\alpha+\beta)}}{\Gamma(\alpha+1)}\left(\frac{1}{\Gamma(\beta+1)}-\frac{B(\alpha+1, \beta)}{\Gamma(\beta)}\right) \\
& +\frac{C_{1} b T^{(\alpha+\beta+1)}}{\Gamma(\alpha+2)}\left(\frac{1}{\Gamma(\beta+1)}-\frac{B(\alpha+2, \beta)}{\Gamma(\beta)}\right)
\end{aligned}
$$

( $\beta$ is considered as integral memory index)
Therefore, the total average cost per unit time per cycle is,

$$
\begin{aligned}
T C_{\alpha, \beta}^{a v}= & \frac{\left(U Q+H C_{\alpha, \beta}(T)+C_{3}\right)}{T} \\
= & \left(\begin{array}{l}
\frac{C_{1} b}{\Gamma(\alpha+2)}\left(\frac{1}{\Gamma(\beta+1)}-\frac{B(\alpha+2, \beta)}{\Gamma(\beta)}\right) T^{\alpha+\beta} \\
+\frac{C_{1} a}{\Gamma(\alpha+1)}\left(\frac{1}{\Gamma(\beta+1)}-\frac{B(\alpha+1, \beta)}{\Gamma(\beta)}\right) T^{\alpha+\beta-1} \\
+\frac{a U}{\Gamma(\alpha+1)} T^{\alpha-1}+\frac{b U}{\Gamma(\alpha+2)} T^{\alpha}+C_{3} T^{-1}
\end{array}\right)
\end{aligned}
$$

The inventory model can be written as follows

$$
\left\{\begin{aligned}
& \operatorname{Min}_{T C_{\alpha, \beta}^{a v}}^{a v}=\left(\begin{array}{l}
A T^{\alpha-1}+B_{1} T^{\alpha}+C T^{\alpha+\beta-1} \\
+D T^{\alpha+\beta}+E T^{-1}
\end{array}\right) A \\
& \text { Subject to } T \geq 0
\end{aligned}\right.
$$

$$
\begin{aligned}
& A=\frac{a U}{\Gamma(\alpha+1)}, B_{1}=\frac{b U}{\Gamma(\alpha+2)}, \\
& C=\frac{C_{1} a}{\Gamma(\alpha+1)}\left(\frac{1}{\Gamma(\beta+1)}-\frac{B(\alpha+1, \beta)}{\Gamma(\beta)}\right) \\
& D=\frac{C_{1} b}{\Gamma(\alpha+2)}\left(\frac{1}{\Gamma(\beta+1)}-\frac{B(\alpha+2, \beta)}{\Gamma(\beta)}\right), E=C_{3}
\end{aligned}
$$

Next, we have discussed the fuzzyfication of the fractional order inventory model

\section{FUZZIFIED OF FRACTIONAL ORDER INVENTORY MODEL}

The fuzzyfication is followed from [22].The related fuzzy inventory model(classical) is described in[18,22].

Here, we fuzzify the cost parameters per unit $\operatorname{cost}(U)$, holding $\operatorname{cost}\left(C_{1}\right)$, setup $\operatorname{cost}\left(C_{3}\right)$ as follows

$$
\begin{aligned}
U & =\left(a_{1}, b_{1}, c_{1}\right), C_{1}=\left(a_{2}, b_{2}, c_{2}\right), C_{3}=\left(a_{3}, b_{3}, c_{3}\right) \\
T C_{\alpha, \beta}^{a v} & =\frac{\left(U Q+H C_{\alpha, \beta}(T)+C_{3}\right)}{T} \\
& =\left(\begin{array}{l}
\frac{\left(a_{2}, b_{2}, c_{2}\right) b}{\Gamma(\alpha+2)}\left(\frac{1}{\Gamma(\beta+1)}-\frac{B(\alpha+2, \beta)}{\Gamma(\beta)}\right) T^{\alpha+\beta} \\
+\frac{\left(a_{2}, b_{2}, c_{2}\right) a}{\Gamma(\alpha+1)}\left(\frac{1}{\Gamma(\beta+1)}-\frac{B(\alpha+1, \beta)}{\Gamma(\beta)}\right) T^{\alpha+\beta-1} \\
+\frac{a\left(a_{1}, b_{1}, c_{1}\right)}{\Gamma(\alpha+1)} T^{\alpha-1}+ \\
\frac{b\left(a_{1}, b_{1}, c_{1}\right)}{\Gamma(\alpha+2)} T^{\alpha}+\left(a_{3}, b_{3}, c_{3}\right) T^{-1}
\end{array}\right)
\end{aligned}
$$

I.J. Intelligent Systems and Applications, 2019, 11, 58-68 


$$
\left.\begin{array}{rl}
T C_{\alpha, \beta, 1}^{a v}= & \left.\begin{array}{l}
\frac{\left(a_{2}\right) b}{\Gamma(\alpha+2)}\left(\frac{1}{\Gamma(\beta+1)}-\frac{B(\alpha+2, \beta)}{\Gamma(\beta)}\right) T^{\alpha+\beta} \\
+\frac{\left(a_{2}\right) a}{\Gamma(\alpha+1)}\left(\frac{1}{\Gamma(\beta+1)}-\frac{B(\alpha+1, \beta)}{\Gamma(\beta)}\right) T^{\alpha+\beta-1} \\
+\frac{a\left(a_{1}\right)}{\Gamma(\alpha+1)} T^{\alpha-1}+\frac{b\left(a_{1}\right)}{\Gamma(\alpha+2)} T^{\alpha}+\left(a_{3}\right) T^{-1}
\end{array}\right), \\
T C_{\alpha, \beta, 2}^{a v}= & \left.\begin{array}{l}
\frac{\left(b_{2}\right) b}{\Gamma(\alpha+2)}\left(\frac{1}{\Gamma(\beta+1)}-\frac{B(\alpha+2, \beta)}{\Gamma(\beta)}\right) T^{\alpha+\beta} \\
+\frac{\left(b_{2}\right) a}{\Gamma(\alpha+1)}\left(\frac{1}{\Gamma(\beta+1)}-\frac{B(\alpha+1, \beta)}{\Gamma(\beta)}\right) T^{\alpha+\beta-1} \\
+\frac{a\left(b_{1}\right)}{\Gamma(\alpha+1)} T^{\alpha-1}+\frac{b\left(b_{1}\right)}{\Gamma(\alpha+2)} T^{\alpha}+\left(b_{3}\right) T^{-1}
\end{array}\right) \\
T C_{\alpha, \beta, 3}^{a v}=\left(\begin{array}{l}
\frac{\left(c_{2}\right) b}{\Gamma(\alpha+2)}\left(\frac{1}{\Gamma(\beta+1)}-\frac{B(\alpha+2, \beta)}{\Gamma(\beta)}\right) T^{\alpha+\beta} \\
+\frac{\left(c_{2}\right) a}{\Gamma(\alpha+1)}\left(\frac{1}{\Gamma(\beta+1)}-\frac{B(\alpha+1, \beta)}{\Gamma(\beta)}\right) T^{\alpha+\beta-1} \\
+\frac{a\left(c_{1}\right)}{\Gamma(\alpha+1)} T^{\alpha-1}+\frac{b\left(c_{1}\right)}{\Gamma(\alpha+2)} T^{\alpha}+\left(c_{3}\right) T^{-1}
\end{array}\right.
\end{array}\right),
$$

\section{A. Signed distance method}

Next, we have discussed the signed distance method its corresponding inventory model.

Fractional order inventory model has been defuzzified using signed distance method[22].

Using signed distance method total average cost is written as follows

$$
\begin{aligned}
& U=\left(a_{1}, b_{1}, c_{1}\right), C_{1}=\left(a_{2}, b_{2}, c_{2}\right), C_{3}=\left(a_{3}, b_{3}, c_{3}\right) \\
& T C_{\alpha, \beta}^{a V S}=\frac{1}{4}\left(T C_{1}+2 T C_{2}+T C_{3}\right) \\
& T C_{\alpha, \beta}^{a V S}=\left(\begin{array}{l}
\frac{b\left(a_{2}+2 b_{2}+c_{2}\right)}{4 \Gamma(\alpha+2)}\left(\frac{1}{\Gamma(\beta+1)}-\frac{B(\alpha+2, \beta)}{\Gamma(\beta)}\right) T^{\alpha+\beta} \\
+\frac{a\left(a_{2}+2 b_{2}+c_{2}\right)}{4 \Gamma(\alpha+1)}\left(\frac{1}{\Gamma(\beta+1)}-\frac{B(\alpha+1, \beta)}{\Gamma(\beta)}\right) T^{\alpha+\beta-1} \\
+\frac{a\left(a_{1}+2 b_{1}+c_{1}\right)}{4 \Gamma(\alpha+1)} T^{\alpha-1}+\frac{b\left(a_{1}+2 b_{1}+c_{1}\right)}{4 \Gamma(\alpha+2)} T^{\alpha}+\frac{\left(a_{3}+2 b_{3}+c_{3}\right)}{4} T^{-1}
\end{array}\right)
\end{aligned}
$$

In this case,Inventory model can be written as follows

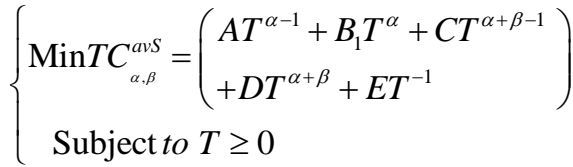

$$
\begin{aligned}
& A=\frac{a\left(a_{1}+2 b_{1}+c_{1}\right)}{4 \Gamma(\alpha+1)}, B_{1}=\frac{b\left(a_{1}+2 b_{1}+c_{1}\right)}{4 \Gamma(\alpha+2)}, \\
& C=\frac{a\left(a_{2}+2 b_{2}+c_{2}\right)}{4 \Gamma(\alpha+1)}\left(\frac{1}{\Gamma(\beta+1)}-\frac{B(\alpha+1, \beta)}{\Gamma(\beta)}\right), \\
& D=\frac{b\left(a_{2}+2 b_{2}+c_{2}\right)}{4 \Gamma(\alpha+2)}\left(\frac{1}{\Gamma(\beta+1)}-\frac{B(\alpha+2, \beta)}{\Gamma(\beta)}\right), E=\frac{\left(a_{3}+2 b_{3}+c_{3}\right)}{4}
\end{aligned}
$$

\section{B. Graded mean integration method}

Fractional order inventory model has been defuzzified using graded mean integration method[22].

Using graded mean integration method, total average cost is written as follows

$$
\begin{aligned}
& U=\left(a_{1}, b_{1}, c_{1}\right), C_{1}=\left(a_{2}, b_{2}, c_{2}\right), C_{3}=\left(a_{3}, b_{3}, c_{3}\right) \\
& T C_{\alpha, \beta}^{a v G}=\frac{1}{6}\left(T C_{\alpha, \beta, 1}^{a v}+4 T C_{\alpha, \beta, 2}^{a v}+T C_{\alpha, \beta, 3}^{a v}\right) \\
& T C_{\alpha, \beta}^{a v G}=\left(\begin{array}{l}
\frac{b\left(a_{2}+4 b_{2}+c_{2}\right)}{6 \Gamma(\alpha+2)}\left(\frac{1}{\Gamma(\beta+1)}-\frac{B(\alpha+2, \beta)}{\Gamma(\beta)}\right) T^{\alpha+\beta} \\
+\frac{a\left(a_{2}+4 b_{2}+c_{2}\right)}{6 \Gamma(\alpha+1)}\left(\frac{1}{\Gamma(\beta+1)}-\frac{B(\alpha+1, \beta)}{\Gamma(\beta)}\right) T^{\alpha+\beta-1} \\
+\frac{a\left(a_{1}+4 b_{1}+c_{1}\right)}{6 \Gamma(\alpha+1)} T^{\alpha-1}+\frac{b\left(a_{1}+4 b_{1}+c_{1}\right)}{6 \Gamma(\alpha+2)} T^{\alpha}+\frac{\left(a_{3}+4 b_{3}+c_{3}\right)}{6} T^{-1}
\end{array}\right)
\end{aligned}
$$

In this case, Inventory model can be written as follows

$$
\left\{\begin{array}{l}
\operatorname{Min} T C_{\alpha, \beta}^{a v G}=\left(\begin{array}{l}
A T^{\alpha-1}+B_{1} T^{\alpha}+C T^{\alpha+\beta-1} \\
+D T^{\alpha+\beta}+E T^{-1}
\end{array}\right) \\
\text { Subject to } T \geq 0
\end{array}\right.
$$

$$
\begin{aligned}
& A=\frac{a\left(a_{1}+4 b_{1}+c_{1}\right)}{6 \Gamma(\alpha+1)}, B_{1}=\frac{b\left(a_{1}+4 b_{1}+c_{1}\right)}{6 \Gamma(\alpha+2)}, \\
& C=\frac{a\left(a_{2}+4 b_{2}+c_{2}\right)}{6 \Gamma(\alpha+1)}\left(\frac{1}{\Gamma(\beta+1)}-\frac{B(\alpha+1, \beta)}{\Gamma(\beta)}\right), \\
& D=\frac{b\left(a_{2}+4 b_{2}+c_{2}\right)}{6 \Gamma(\alpha+2)}\left(\frac{1}{\Gamma(\beta+1)}-\frac{B(\alpha+2, \beta)}{\Gamma(\beta)}\right), E=\frac{\left(a_{3}+4 b_{3}+c_{3}\right)}{6}
\end{aligned}
$$

\section{NUMERICAL EXAMPLE}

To illustrate numerically of the developed fractional order inventory model, we consider empirical values of the various parameters in proper units as $\gamma=45,\left(a_{1}, b_{1}, c_{1}\right)=(12,14,16)$, $\left(a_{2}, b_{2}, c_{2}\right)=(19,20,24),\left(a_{3}, b_{3}, c_{3}\right)=(280,300,310){ }^{\text {and }}$ required solution has been made using matlab minimization method.

where 
A. Optimal ordering interval and Minimized total average cost using signed distance method.

Table 2. Optimal ordering interval and minimized total average cost using signed distance method where $0<\alpha \leq 1.0, \beta=1.0$ described in sectionVII.

\begin{tabular}{|c|c|c|c|}
\hline$\alpha$ & $\beta$ & $T_{\alpha, \beta}^{s^{*}}$ & $T C_{\alpha, \beta}^{s^{*}}$ \\
\hline 0.1 & 1.0 & $\mathbf{1 . 6 8 1 1}$ & $1.4979 \times 10^{3}$ \\
\hline 0.2 & 1.0 & 1.4845 & $1.6404 \times 10^{3}$ \\
\hline $\begin{array}{c}0.3 \text { (growing } \\
\text { memory effect) }\end{array}$ & 1.0 & 1.3033 & $1.7508 \times 10^{3}$ \\
\hline 0.4 & 1.0 & 1.1396 & $1.8238 \times 10^{3}$ \\
\hline $\mathbf{0 . 5}$ & 1.0 & 0.9952 & $\begin{array}{c}\mathbf{1 . 8 5 7 0 \times 1 0}(\mathbf{m a x} \\
\mathbf{i m u m})\end{array}$ \\
\hline 0.6 & 1.0 & 0.8722 & $1.8518 \times 10^{3}$ \\
\hline 0.7 & 1.0 & 0.7714 & $1.8129 \times 10^{3}$ \\
\hline $0.8 \uparrow$ & 1.0 & 0.6927 & $1.7480 \times 10^{3}$ \\
\hline 0.9 & 1.0 & 0.6341 & $1.6653 \times 10^{3}$ \\
\hline $\mathbf{1 . 0}$ & 1.0 & 0.5927 & $1.5731 \times 10^{3}$ \\
\hline
\end{tabular}

From table 2, it is found that for $\alpha=0.5$ minimized total average cost is maximum then gradually falls down. Hence, suddenly, business policy falls down then gradually improves. In general, in reality it happens always i.e. profit increase or decrease with respect to its previous effect.

Table 3. Optimal ordering interval and minimized total average cost using signed distance method for $0<\beta \leq 1.0, \alpha=1.0$ described in section-VII-A.

\begin{tabular}{|c|c|c|c|}
\hline$\alpha$ & $\beta$ & $T_{\alpha, \beta}^{{ }^{*}}$ & $T C_{\alpha, \beta}^{{ }^{*}}$ \\
\hline 1.0 & 0.1 & $\mathbf{1 . 1 5 3 3 9}$ (maximum) & $1.2231 \times 10^{3}$ \\
\hline 1.0 & 0.2 & 1.0112 & $1.3404 \times 10^{3}$ \\
\hline 1.0 & 0.3 & 0.8928 & $1.4341 \times 10^{3}$ \\
\hline 1.0 & 0.4 & 0.8007 & $1.5035 \times 10^{3}$ \\
\hline 1.0 & 0.5 & 0.7318 & $1.5505 \times 10^{3}$ \\
\hline 1.0 & 0.6 & 0.6814 & $1.5788 \times 10^{3}$ \\
\hline 1.0 & 0.7 & 0.6454 & $1.5918 \times 10^{3}$ \\
\hline 1.0 & $\mathbf{0 . 8} \uparrow$ & $\mathbf{0 . 6 2 0 2}$ & $\mathbf{1 . 5 9 3 4 \times 1 0}(\mathbf{m a x i m u m})$ \\
\hline 1.0 & 0.9 & 0.6033 & $1.5836 \times 10^{3}$ \\
\hline 1.0 & $\mathbf{1 . 0}$ & 0.5927 & $1.5731 \times 10^{3}$ \\
\hline
\end{tabular}

From table-3, it is clear that in absence of differential memory index in presence of integral memory index, minimized total average cost is maximum at $\beta=0.8$ then gradually falls down above and below. Hence, suddenly, business policy falls down then gradually improves. In absence of differential memory index, minimized total average cost is low compared to the presence of differential memory index.

Table 4 shows that in presence of integral memory index with varying differential memory index, minimized total average cost is very much low compared to the two cases (i) presence of integral memory index in absence of differential memory index,(ii) presence of differential memory index in absence of integral memory index.
Table 4. $T_{\alpha, \beta}^{s^{*}}$ and $T C_{\alpha, \beta}^{s^{*}}$ using signed distance method where $0<\alpha \leq 1.0, \beta=0.5$ described in section-VII-A.

\begin{tabular}{|c|c|c|c|}
\hline$\alpha$ & $\beta$ & $T_{\alpha, \beta}^{s^{*}}$ & $T C_{\alpha, \beta}^{{ }^{*}}$ \\
\hline 0.1 & 0.5 & $\mathbf{3 . 5 4 4 3}$ & $1.1330 \times 10^{3}$ \\
\hline 0.2 & 0.5 & 2.8779 & $1.2873 \times 10^{3}$ \\
\hline 0.3 & 0.5 & 2.3685 & $1.4256 \times 10^{3}$ \\
\hline 0.4 & 0.5 & 1.9601 & $1.5406 \times 10^{3}$ \\
\hline 0.5 & 0.5 & 1.6251 & $1.6256 \times 10^{3}$ \\
\hline 0.6 & 0.5 & 1.3495 & $1.6757 \times 10^{3}$ \\
\hline $\mathbf{0 . 7}$ & 0.5 & 1.1267 & $\mathbf{1 . 6 8 9 3 \times 1 0}(\mathbf{m a x i m u m})$ \\
\hline $0.8 \uparrow($ growing & 0.5 & 0.9529 & $1.6686 \times 10^{3}$ \\
\hline memory effect) & 0.5 & 0.8235 & $1.6196 \times 10^{3}$ \\
\hline 0.9 & 0.5 & 0.7318 & $1.5505 \times 10^{3}$ \\
\hline $\mathbf{1 . 0}$ & & & \\
\hline
\end{tabular}

From the above table 2-4 where minimized total average cost and optimal ordering interval are evaluated using signed distance method, the conclusions are done as

(i) In presence of differential memory index in absence of integral memory index, for $\alpha=0.5$ minimized total average cost is maximum then gradually falls down. Hence, suddenly, business policy falls down then gradually improves.

(ii) In absence of differential memory index with presence of integral memory index, minimized total average cost is maximum at $\beta=0.8$ then gradually falls down above and below. Hence, suddenly, business policy falls down then gradually improves. In absence of differential memory index, minimized total average cost is low compared to the presence of differential memory index.

(iii) In presence of integral memory index with varying differential memory index, minimized total average cost is very much low compared to the two cases (i) presence of integral memory index with absence of differential memory index,(ii) presence of differential memory index with absence of integral memory index.

B. Optimal ordering interval and minimized total average cost using graded mean integration method

Table 5. Optimal ordering interval and minimized total average cost using graded mean integration method where $0<\alpha \leq 1, \beta=1.0$.

\begin{tabular}{|c|c|c|c|}
\hline$\alpha$ & $\beta$ & $T_{\alpha, \beta}^{G^{*}}$ & $T C_{\alpha, \beta}^{G^{*}}$ \\
\hline 0.1 & 1.0 & $\mathbf{1 . 6 9 1 7}$ & $1.4917 \times 10^{3}$ \\
\hline 0.2 & 1.0 & 1.4937 & $1.6338 \times 10^{3}$ \\
\hline $\begin{array}{c}0.3 \text { (growing } \\
\text { memory effect) }\end{array}$ & 1.0 & 1.3114 & $1.744 \times 10^{3}$ \\
\hline 0.4 & 1.0 & 1.1466 & $1.8171 \times 10^{3}$ \\
\hline 0.5 & 1.0 & 1.0014 & $\begin{array}{c}\mathbf{1 . 8 5 0 8 \times 1 0}(\mathbf{m a x i} \\
\text { mum) }\end{array}$ \\
\hline 0.6 & 1.0 & 0.8776 & $1.8462 \times 10^{3}$ \\
\hline 0.7 & 1.0 & 0.7762 & $1.8081 \times 10^{3}$ \\
\hline $0.8 \uparrow$ & 1.0 & 0.6969 & $1.7439 \times 10^{3}$ \\
\hline 0.9 & 1.0 & 0.6378 & $1.6620 \times 10^{3}$ \\
\hline $\mathbf{1 . 0}$ & 1.0 & 0.5960 & $1.5704 \times 10^{3}$ \\
\hline
\end{tabular}


From table 5, table 2, it is observed that minimized total average cost using graded integration method is low compared to the signed distance method.Table-5 also shows that at $\alpha=0.5$, minimized total average cost becomes maximum then gradually decreases below and above. Profit once, loses then gradually increases below and above. Optimal ordering interval gradually increases with respect to memory index.

Table 6. $T_{\alpha, \beta}^{G^{*}}$ and $T C_{\alpha, \beta}^{G^{*}}$ using graded mean integration method where $0<\beta \leq 1.0, \alpha=1.0$ described in section-VII.

\begin{tabular}{|c|c|c|c|}
\hline$\alpha$ & $\beta$ & $T_{\alpha, \beta}^{G^{*}}$ & $T C_{\alpha, \beta}^{G^{*}}$ \\
\hline 1.0 & 0.1 & $\mathbf{1 . 1 5 6 4}$ & $1.2222 \times 10^{3}$ \\
\hline 1.0 & 0.2 & 1.0151 & $1.3384 \times 10^{3}$ \\
\hline 1.0 & 0.3 & 0.8970 & $1.4312 \times 10^{3}$ \\
\hline 1.0 & 0.4 & 0.8050 & $1.5001 \times 10^{3}$ \\
\hline 1.0 & 0.5 & 0.7359 & $1.5470 \times 10^{3}$ \\
\hline 1.0 & 0.6 & 0.6854 & $1.5752 \times 10^{3}$ \\
\hline 1.0 & $\mathbf{0 . 7}$ & 0.6491 & $1.5884 \times 10^{3}$ \\
\hline 1.0 & $0.8 \uparrow$ (growing & 0.6237 & $\mathbf{1 . 5 9 0 2 \times 1 0 ^ { 3 }}$ (maximum) \\
\hline 1.0 & 0.9 & 0.6067 & $1.5834 \times 10^{3}$ \\
\hline 1.0 & $\mathbf{1 . 0}$ & 0.5960 & $1.5704 \times 10^{3}$ \\
\hline
\end{tabular}

From table 6, table 3, it is clear that numerical value of the minimized total average cost using graded integration method is low compared to the signed distance method.

Table 7. $T_{\alpha, \beta}^{G^{*}}$ and $T C_{\alpha, \beta}^{G^{*}}$ using graded mean integration method where $0<\alpha \leq 1, \beta=0.5$ described in section-VII.

\begin{tabular}{|c|c|c|c|}
\hline$\alpha$ & $\beta$ & $T_{\alpha, \beta}^{G^{*}}$ & $T C_{\alpha, \beta}^{G^{*}}$ \\
\hline 0.1 & 0.5 & $\mathbf{3 . 5 7 0 8}$ & $1.1277 \times 10^{3}$ \\
\hline 0.2 & 0.5 & 2.8972 & $1.2816 \times 10^{3}$ \\
\hline 0.3 & 0.5 & 2.3832 & $1.4196 \times 10^{3}$ \\
\hline 0.4 & 0.5 & 1.9718 & $1.5344 \times 10^{3}$ \\
\hline 0.5 & 0.5 & 1.6346 & $1.6194 \times 10^{3}$ \\
\hline 0.6 & 0.5 & 1.3574 & $1.6698 \times 10^{3}$ \\
\hline $\mathbf{0 . 7}$ & 0.5 & 1.1333 & $\mathbf{1 . 6 8 3 9} \times 10^{3}$ \\
\hline $\begin{array}{c}0.8 \uparrow \text { (growing } \\
\text { memory effect) }\end{array}$ & 0.5 & 0.9585 & $1.6637 \times 10^{3}$ \\
\hline 0.9 & 0.5 & 0.8283 & $1.6154 \times 10^{3}$ \\
\hline $\mathbf{1 . 0}$ & 0.5 & 0.7359 & $1.5470 \times 10^{3}$ \\
\hline
\end{tabular}

From table 7 and 4, it is found that numerical values of the minimized total average cost using graded integration method is low compared to the signed distance method.

From all observations of the numerical examples, it is observed that numerical values of the minimized total average cost using graded integration method is low compared to the signed distance method.

Here, we want to include a real world example where, this mathematical results can knock. All numerical examples clear that at once profit is losses then gradually increases. In reality, in the village- area, selling rate of shoes, cosmetic products, clothes increase in the season wise in the different festivals of the local area. For that reason, profit of the business increases or decreases. Due to favorable or unfavorable social, environment, cost parameters are highly uncertain. To eradicate this uncertainty, fuzzy cost parameters are incorporated.

In the next section, we have discussed the pictorial behavior of the total average cost function with respect to different memory indexes and inventory level, inventory holding cost for different memory index with respect to time.

\section{GRAPHICAL PRESENTATIONS}

Scatter diagram of the minimized total average cost and optimal ordering interval using the above tables.

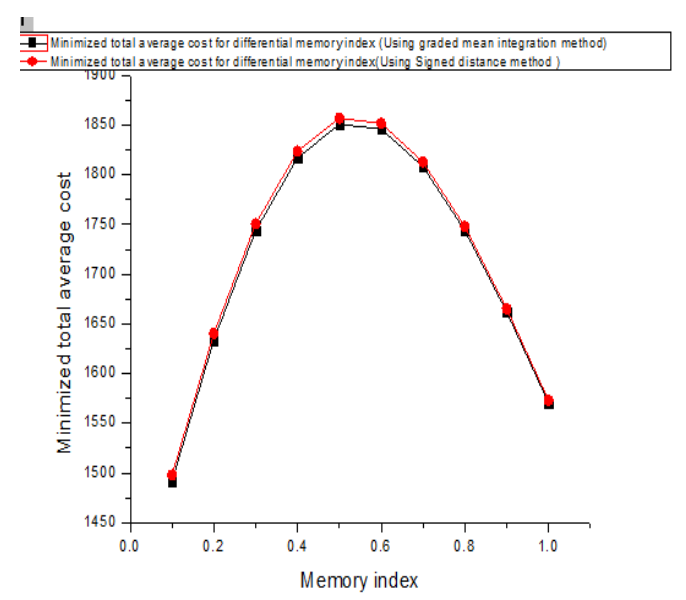

Fig.1. Comparison of the minimized total average cost with respect to differential memory index using (i) graded mean integration method (ii) signed distance method.

From fig.1, it is clear that the graph of the minimized total average cost is parabolic type whose vertex is upward. On the other hand, it is also clear that, the numerical values using graded mean integration method is low compared to the signed distance method.

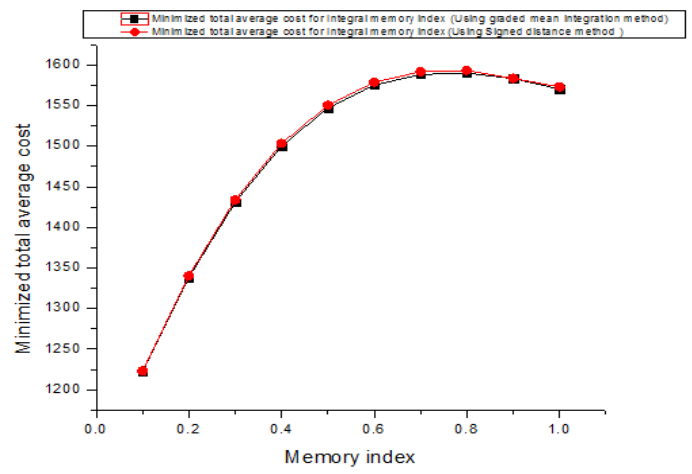

Fig.2. Comparison of the minimized total average cost with respect to Integral memory index corresponding (i) graded mean integration method (ii) signed distance method.

From fig.2, it is found that the minimized total average cost function is not exactly parabolic but approximately. On the other hand, it is also clear that, the numerical values using graded mean integration method is low compared to the signed distance method but their difference is very negligible. 


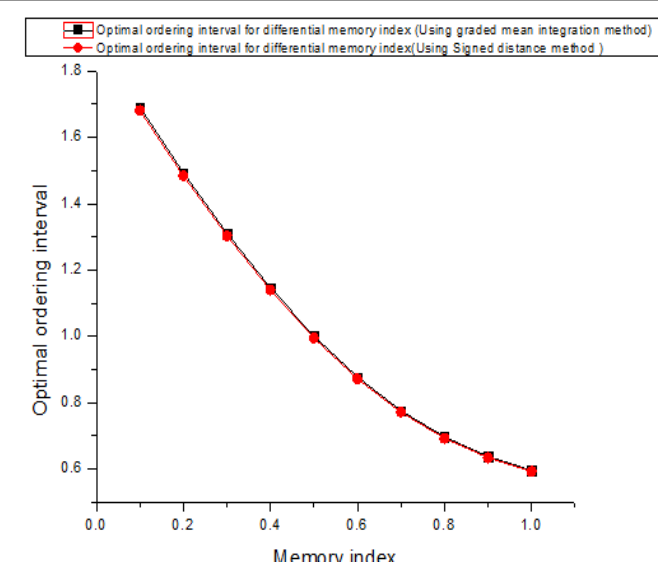

Fig.3. Comparison of the Optimal ordering interval with respect to differential memory index (i) graded mean integration method (ii) signed distance method.

From fig. 3 , it is found that the minimized total average cost function is exponential type. On the other hand, it is also clear that, the numerical values of the optimal ordering interval using graded mean integration method is high compared to the signed distance method but their difference is very negligible.

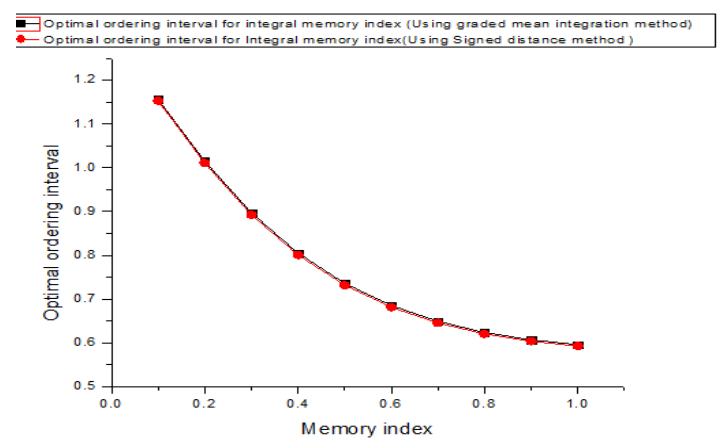

Fig.4. Comparison of the optimal ordering interval with respect to Integral memory index corresponding (i) graded mean integration method (ii) signed distance method respectively.

From fig. $3 \& 4$, it is observed that the minimized total average cost is exponential type. But the numerical values of the optimal ordering interval for presence of differential memory index is high compared to the absence of differential memory index i.e. in presence of integral memory index.

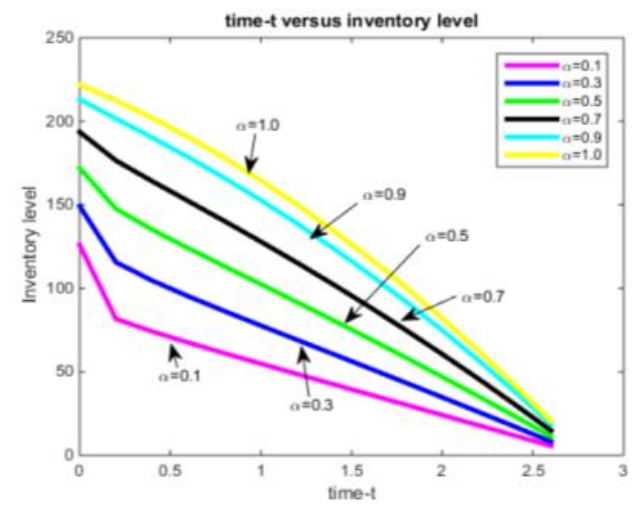

Fig.5. Time-t versus Inventory level for different values of $\alpha$.
From fig.5, it is clear that qualitatively different behavior of inventory level is found for(i) long memory effect,(ii) short memory effect,(iii) memory less system. Rate of change of inventory level for long memory effect is concave type but in short memory effect, is not concave type.

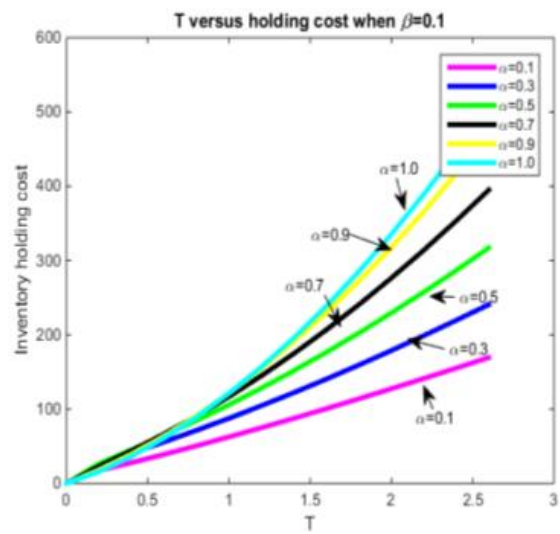

(a)

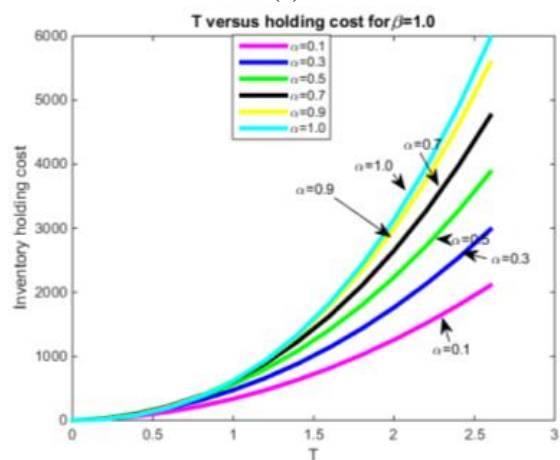

(b)

Fig.6. (a-b)Inventory holding cost versus ordering interval- $T$ for different values of $\alpha, \beta$.

From fig.6.(a-b), it is observed that for gradually increasing memory effect(here, memory effect quantity $\alpha$ ) holding cost is gradually decreasing for different values of $\beta$. From the fig.6, it is observed that initially lowest inventory holding cost is at $\alpha=0.1$ then gradually increases highest inventory holding cost is at $\alpha=0.1$.

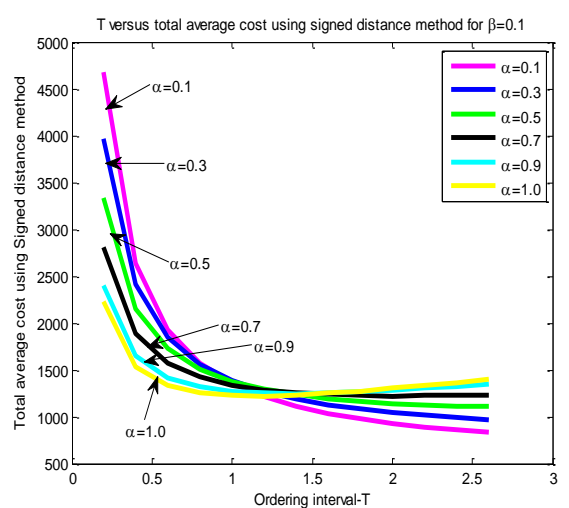

(a)

Fig.7. (a)ordering interval- $T$ - Total average cost using signed distance method for different values of $\alpha, \beta$. 
From the fig.7.(a) we can conclude that total average cost using signed distance method is concave type. Total average cost lowest at the $\alpha=0.1$ with respect to ordering interval as well as total average cost reaches highest level for lowest ordering interval at $\alpha=0.1$.

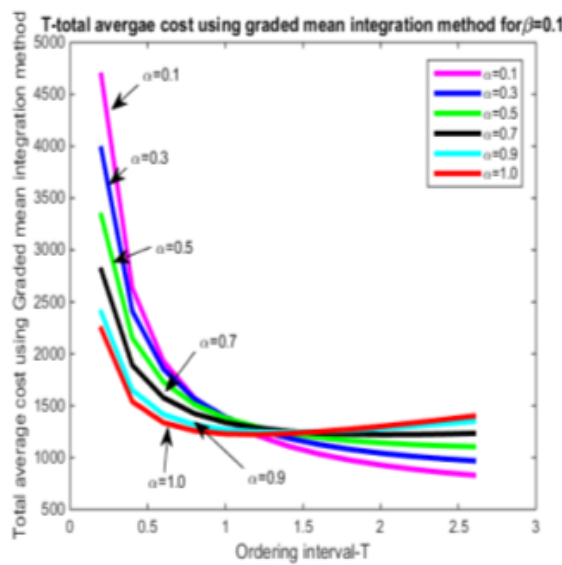

(a)

Fig.8. (a)Ordering interval- $T$ - total average cost using graded mean integration method for different values of $\alpha, \beta$.

In fig-8(a), we want to show the behavior of total average cost function with respect to the ordering interval for different memory index.

From all fig.8.(a)it is observed that total average cost becomes low for $\alpha=0.1$.

\section{CONCLUSION}

In this paper, some useful ideas were presented to deal with fractional order inventory model with fuzzy environment. In economic model memory effect should be incorporated due to the fact that some economic facts depend on the past experience such as environment, social situation, product quality can increase or decrease the selling rate of the product. Fuzzyfied can close to the reality to incorporate the uncertainty behavior of some economic parameters like set up cost, holding cost, per unit cost. We have also analyzed the fractional order inventory model under fuzzy environment to evaluate the optimum results of ordering interval and total average cost.Fig.5, 6,8helps to conclude that the neglect of memory effects in the economic model may lead to qualitatively different output of inventory leveland holding cost, minimized total average cost.Numerical examples provide us that minimized total average cost using graded integration method is low compared to the signed distance method. In long memory effect profit is maximum compared to the low memory effect as well as memory less system for both methods (i) graded mean integration method,(ii) signed distance method. This paper helps to come out the developed thought of classical fuzzy inventory model. This paper also helps to include reality of the system using the memory effect property of the system. More work on the inventory system can be done under the fuzzy environment using fractional calculus for the future research work.

\section{ACKNOWLEDGEMENTS}

Authors would like to express their sincere thanks to the referees and editors for their valuable comments and suggestions for improving the paper. The first author would also like to thank the Department of science and Technology, Government of India, New Delhi, for the financial assistance under AORC, Inspire fellowship Scheme towards this research work.

\section{REFRENCES}

[1] M.Saeedian, M. Khalighi, N. Azimi-Tafreshi, G. R. Jafari, M. Ausloos(2017), Memory effects on epidemic evolution: The susceptible-infected-recovered epidemic model, Physical Review E95,022409.

[2] V.V.Tarasova, V.E.Tarasov., (2016) "Memory effects in hereditary Keynesian model" Problems of Modern Science and Education.No.38 (80). P. 38-44. DOI: 10.20861/2304-2338-2016-80-001 [in Russian].

[3] V.V, Tarasova, V.E.Tarasov. A generalization of the concepts of the accelerator and multiplier to take into account of memory effects in macroeconomics//Ekonomika I Predprinmatelstvo [Journal of Economy and Entrepreneurship], 2016.vol.10.No.10-3.P.[12]-1129.[in Russian].

[4] V.V, Tarasova, V.E.Tarasov. Marginal utility for economic processes with memory//AlmanahSovremennojNauki I Obrazovaniya [Almanac of Modern Science and Education], 2016.No.7(109).P.108-113[in Russian].

[5] V.V, Tarasova, V.E.Tarasov. Fractional Dynamics of Natural Growth and Memory Effect in Economics,European Research.2016.No.12(23).P.30-37.

[6] V.E.Tarasov, V.V.Tarasova., (2016)." Long and short memory in economics: fractional-order difference and differentiation "IRA-International Journal of Management and Social Sciences. Vol. 5.No. 2. P. 327-334. DOI: 10.21013/jmss.v5.n2.p10.

[7] V.E.Tarasov, V.V.Tarasova., (2017)."Economic interpretation of fractional derivatives". Progress in Fractional Differential and Applications.3.No.1, 1-6.

[8] T.Das, U.Ghosh, S.Sarkar and S.Das,(2018)" Time independent fractional Schrodinger equation for generalized Mie-type potential in higher dimension framed with Jumarie type fractional derivative". Journal of Mathematical Phy sics,59, 022111; doi: 10.1063/1.4999262.

[9] R.Pakhira, U.Ghosh, S.Sarkar., (2018) Study of Memory Effects in an Inventory Model Using Fractional Calculus, Applied Mathematical Sciences, Vol. 12, no. 17, 797 824.

[10] R.Pakhira, U.Ghosh, S.Sarkar., "Application of Memory effects In an Inventory Model with Linear Demand and No shortage", International Journal of Research in Advent Technology, Vol.6, No.8, 2018.

[11] R.Pakhira., U.Ghosh., S.Sarkar.,(2018).Study of Memory Effect in an Inventory Model with Linear Demand and Salvage Value, International Journal of Applied Engineering ResearchISSN 0973-4562 Volume 13, Number 20 (2018) pp. 14741-14751.

[12] K.S.Miller, B.Ross., (1993).“An Introduction to the Fractional Calculus and Fractional Differential Equations".JohnWiley\&Sons, New York, NY, USA. 
[13] I.Podubly(1999), "Fractional Differential Equations, Mathematics in Science and Engineering", Academic Press, San Diego, Calif,USA.198.

[14] M.Caputo.,(1967).Linear models of dissipation whose frequency independent, "Geophysical Journal of the Royal Astronomical Society. 13(5), 529-539.

[15] U.Ghosh, S. Sengupta, S.Sarkar, S.Das., 2015. Analytic Solution of linear fractional differential equation with Jumarie derivative in term of Mittag-Leffler function .American Journal of Mathematical Analysis 3(2).32-38

[16] P.Majumder,U.K.Bera, and M.Maiti., An EPQ modelof deteriorating items under partial trade credit financing and demand declining market in crisp and fuzzy environment, Procedia Computer Science, 45 (2015), 780-789.

[17] N. K. Mandal, Fuzzy economic order quantity modelwith ranking fuzzy number cost parameters, Yugoslav Journal of Operations Research, 22 (2012), 247-264.

[18] D. Sharmita and R.Uthayakumar,'Inventory model for deteriorating items involving fuzzy with shortages and exponential demand" International Journal of Supply and operations management, Nov 2015,volume 2,Issue 3, pp.888-904

[19] G. Rotundo, in Logistic Function in Large Financial Crashes, The Logistic Map and the Route to Chaos: From the Beginning to Modern Applications, edited by $\mathrm{M}$. Ausloos and M. Dirickx(Springer-Verlag, Berlin/Heidelberg, 2005), pp. 239-258.

[20] R.Pakhira, U.Ghosh, S.Sarkar.,(2019). Study of Memory Effect in an Inventory Model with Quadratic Type Demand Rate and Salvage Value, Applied Mathematical Sciences, Vol. 13, 2019, no. 5, 209 - 223.

[21] Tarasov.V.E, Tarasova.V.V, Elasticity for economic processes with memory: fractional differential calculus approach, Fractional Differential Calculus, Vol-6, No2(2016),219-232.

[22] S.Saha, T. Chakrabarti,A Fuzzy Inventory Model for Deteriorating Items with Linear Price Dependent Demand in a Supply Chain,Intern. J. Fuzzy Mathematical Archive, Vol. 13, No. 1, 2017, 59-67, ISSN: 2320 -3242 (P), 2320-3250 (online).

[23] R.Pakhira, U.Ghosh, S.Sarkar., (2019). Application of memory effect in an inventory model with price dependent demand rate during shortage, I.J. Education and Management Engineering, 2019,DOI: 10.5815.

[24] R.Pakhira,U.Ghosh,S.Sarkar.,((2019).Study of Memory Effect In an Inventory Model with Linear Demand and Shortage, International Journal of Mathematical Sciences and Computing(IJMSC), ISSN: 2310-9025(Print), DOI:10.5815/ijmsc.2019.02.05.

\section{Authors' Profiles}

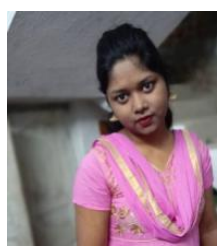

Rituparna Pakhira is currently P.HD DST InspireResearch fellow Department of Applied Mathematics, University of Calcutta, west Bengal India. Kolkata, India.She obtained MSc in Mathematics from Jadavpuruniversity, west Bengal India in 2015.She is mainly interested application of Fractional Calculus different topic of operation research. She has 8 publications in different international Journals.

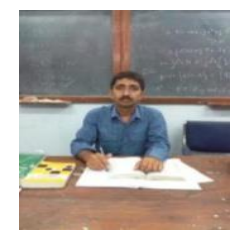

Uttam Ghosh is Assistant Professor of Applied Mathematics in University of Calcutta. His research field includes Fractal geometry, Information theory, Percolation theory, Biomathematics and Fractional Calculus. He has 48 publications in reputed national and international Journals.

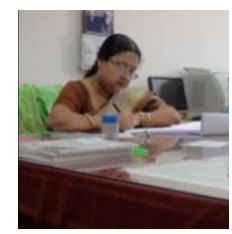

Susmita Sarkar is Professor of Applied Mathematics, University of Calcutta. Her field of research includes Plasma Dynamics, Biomathematics and Fractional Calculus. She has more than 81 research publications in reputed international journals.Dr. SusmitaSarkar was TWAS Associate and ICTP regular Associate.

How to cite this paper: Rituparna Pakhira, Uttam Ghosh, Susmita Sarkar, "Study of Memory Effect in a Fuzzy EOQ Model with No Shortage", International Journal of Intelligent Systems and Applications(IJISA), Vol.11, No.11, pp.58-68, 2019. DOI: $10.5815 /$ ijisa.2019.11.06 